\title{
On Positive Semigroups
}

By

\author{
Derek W. ROBINSON*
}

\begin{abstract}
We prove versions of the Feller-Miyadera-Phillips theorem characterizing the generators of positive $C_{0^{-}}$and $C_{0}^{*}$-semigroups on ordered Banach spaces, for which the norm and dual norm are monotonic. Two proofs are given. The first is based on half-norm theory whilst the second exploits the existence of an equivalent Riesz norm. This latter norm exists if, and only if, the positive cone is normal and generating.
\end{abstract}

\section{§ 0. Introduction}

In a previous paper [1] we developed the theory of generators of positive $C_{0^{-}}$and $C_{0}^{*}$-semigroups acting on ordered Banach spaces equipped with a Riesz norm. The purpose of the present paper is to extend the theory to spaces whose norm and dual norm are both monotonic. This weakening of the underlying assumptions leads to a slight weakening of the conclusions; norm estimates on the semigroups are replaced by estimates of the norms on the positive elements.

There are two methods of extending the Riesz norm results to monotonic norms. The first is direct and is based upon recent characterizations of canonical half-norms [2]. The second is less direct and combines the results of [1] together with the construction of an equivalent Riesz norm. This latter method indicates the necessity of norm-monotonicity in the basic theory of generators of positive semigroups.

We adopt the notation and terminology of [1] [2] throughout the sequel and rely on these papers for background references.

Communicated by H. Araki, February 2, 1983.

* Department of Mathematics, Institute of Advanced Studies, Australian National University, Canberra. 


\section{§1. Positive Semigroups}

Let $\mathfrak{B}(\mathscr{B})$ denote the bounded operators on the ordered Banach space $\left(\mathscr{B}, \mathscr{B}_{+},\|\cdot\|\right)$ and define the mapping $A \in \mathscr{B}(\mathscr{B}) \longmapsto\|A\|_{+} \in \boldsymbol{R}_{+}$ by

$$
\|A\|_{+}=\sup \left\{\|A a\| ; a \in \mathscr{B}_{+} \cap \mathscr{B}_{1}\right\} .
$$

Clearly $\|A\|_{+} \leq\|A\|$, where $\|\cdot\|$ is the usual operator norm. But if $\mathscr{B}_{+}$ is $\gamma$-generating then $\|A\| \leq\left.\gamma\right|^{\prime} A \|_{+}$which implies that $\|\cdot\|_{+}$is a norm on $\mathscr{B}(\mathscr{B})$ and it is equivalent to $\|\cdot\|$. If the norm on $\mathscr{B}$ is a Riesz norm then it follows from Lemma 3.3 of [1] that the two norms $\|\cdot\|$ and $\|\cdot\|_{+}$on $\mathfrak{B}(\mathscr{B})$ are in fact equal.

The principal new result on generators is a version of the FellerMiyadera-Phillips theorem for positive $\|\cdot\|_{+}-$-bounded semigroups. We first give a proof using half-norm theory [2].

Theorem 1.1. Let $H$ be a linear operator on the ordered Banach space $\left(\mathscr{B}, \mathscr{B}_{+},\|\cdot\|\right)$ and let $N$ denote the canonical half-norm associated with $\left(\mathscr{B}_{+},\|\cdot\|\right)$. Assume that $\|\cdot\|$ is monotonic on $\mathscr{B}$ and the dualnorm $\|\cdot\|$ is monotonic on $\mathscr{B}^{*}$.

The following conditions are equivalent:

1. $H$ generates a positive $C_{0}$-semigroup with the bounds

$$
\left\|S_{t}\right\|_{+} \leq M e^{\omega t}, \quad t \geq 0
$$

2. $H$ is norm-densely defined, norm-closed, and satisfies

$$
R(I+\alpha H)=\mathscr{B}
$$

and

$$
N\left((I+\alpha H)^{n} a\right) \geq(1-\alpha \omega)^{n} N(a) / M
$$

for $a \in D\left(H^{n}\right)$ and $n \geq 1$, for all small $\alpha>0$.

Remark. The case $M=1, \omega=0$, corresponds to the Hille-Yosida theorem, i. e., $S$ is $\|\cdot\|_{+}$-contractive. The dissipativity criteria then reduce to the single condition $N((I+\alpha H) a) \geq N(a)$, because the higher order conditions follow by iteration.

Proof. $1 \Rightarrow 2$. All properties except the $N$-dissipativity are standard 
consequences of semigroup theory. To prove the $N$-dissipativity we first remark that monotonicity of $\|\cdot\|$ on $\mathscr{B}^{*}$ is equivalent to

$$
N(a)=\inf \left\{\|b\|_{1} ; b \geq a, b \geq 0\right\}
$$

for all $a \in \mathscr{B}$. This follows from Theorem 2.4 of [2], where monotonicity is referred to as 1-monotonicity. Therefore

$$
N\left(S_{t} a\right)=\inf \left\{|b| \| ; b \geq S_{t} a, b \geq 0\right\} .
$$

Now set $b=S_{t} c$ with $c \geq a$ and $c \geq 0$. One has $b \geq S_{t} a$ and $b \geq 0$ because $S$ is positive. Hence

$$
\begin{aligned}
N\left(S_{t} a\right) & \leq \inf \left\{\left\|S_{t} c\right\| ; c \geq a, c \geq 0\right\} \\
& \leq\left\|S_{t}\right\|_{+} N(a) \leq M e^{\omega t} N(a) .
\end{aligned}
$$

Finally the $N$-dissipativity conditions follow from Laplace transformation and convexity of $N$ as in the proof of Theorem 3.5 of [1].

$2 \Rightarrow 1$. Since $\|\cdot\|$ is monotonic on $\mathscr{B}$ the cone $\mathscr{B}_{+}$is normal. Therefore $\|\cdot\|$ is equivalent to the order-norm $\|\cdot\|_{N}$, where

$$
\|a\|_{N}=N(a) \vee N(-a) \text {. }
$$

But the $N$-dissipativity conditions imply that

$$
\left\|_{i}(I+\alpha H)^{n} a\right\|_{N} \geq(1-\alpha \omega)^{n}|| a \|_{N} / M
$$

for all $a \in D\left(H^{n}\right)$ and $n \geq 1$. Hence it follows from the FellerMiyadera-Phillips theorem that $H$ generates a $C_{0}$-semigroup $S$ satisfying

$$
\left\|S_{t}\right\|_{N} \leq M e^{\omega t} \text {. }
$$

Moreover $S$ is positive by the argument used in the proof of Theorem 3.1 of [1]. Finally since $\|\cdot\|$ is monotonic on $\mathscr{B}$

$$
\|a\|_{N}=N(a)=\|a\|
$$

for all $a \geq 0$, by Theorem 2.3 of [2]. Therefore

$$
\begin{aligned}
\left\|S_{t}\right\|_{+}= & \sup \left\{\left\|S_{t} a\right\| ; a \in \mathscr{B}_{+} \cap \mathscr{B}_{1}\right\} \\
= & \sup \left\{\left\|S_{t} a\right\|_{N} ; a \in \mathscr{B}_{+} \cap \mathscr{B}_{1}\right\} \\
& \leq M e^{\omega t} .
\end{aligned}
$$

Remark. Monotonicity of the norm on $\mathscr{B}^{*}$ was used to prove $1 \Rightarrow 2$, and monotonicity on $\mathscr{B}$ was necessary for $2 \Rightarrow 1$.

Next we derive an analogue of Theorem 1.1 for $C_{0}^{*}$-semigroups. 
Theorem 1.2. Assume that $\left(\mathscr{B}, \mathscr{B}_{+},\|\cdot\|\right)$ is the dual of an ordered Banach space $\left(\mathscr{B}_{*}, \mathscr{B}_{*+}, ! \cdot \cdot \|\right)$ and that the norm is monotonic on both $\mathscr{B}$ and $\mathscr{B}_{*}$. Further let $N$ denote the canonical half-norm associated with $\mathscr{B}_{+}$.

If $H$ is a linear operator on $\mathscr{B}$ the following conditions are equivalent:

1. $H$ generates a positive $C_{0}^{*}$-semigroup satisfying

$$
\left\|S_{t}\right\|_{+} \leq M e^{\omega t},
$$

2. $H$ is weak $k^{*}$-densely defined, weak $k^{*}$-weak $k^{*}$-closed and satisfies

$$
R(I+\alpha H)=\mathscr{B}
$$

and

$$
N\left((I+\alpha H)^{n} a\right) \geq(1-\alpha \omega)^{n} N(a) / M
$$

for $a \in D\left(H^{n}\right)$ and $n \geq 1$, for all small $\alpha>0$.

Proof. The proof follows from a combination of the proof of Theorem 3.4 of [1] together with the proof of Theorem 1.1. The only new feature is the derivation of the bounds on $S$ from Condition 2.

Following the proof of Theorem 3.4 of [1] one constructs a positive $C_{0}$-semigroup $S^{*}$ on $\mathscr{B}_{*}$ and then by the proof of $2 \Rightarrow 1$ in Theorem 1.1 one deduces that

$$
\begin{aligned}
\left\|S_{t}^{*}\right\|_{+}= & \sup \left\{\left\|S_{t}^{*} f\right\| ; f \in \mathscr{B}_{*+} \cap \mathscr{B}_{* 1}\right\} \\
& \leq M e^{\omega t} .
\end{aligned}
$$

The dual semigroup $S$ on $\mathscr{B}$ is then positive, weak*-continuous, and it remains to prove that it satisfies the above bounds. This is established by the following lemma.

Lemma 1.3. Let $A$ be a positive bounded operator on the ordered Banach space $\left(\mathscr{B}, \mathscr{B}_{+},\|\cdot\|\right)$. If $\|\cdot\|$ is monotonic on $\mathscr{B}$ then

$$
\|A\|_{+} \leq\left\|A^{*}\right\|_{+},
$$

with equality if the norm is also monotonic on $\mathscr{B}^{*}$.

Proof. By definition

$$
\|A\|_{+}=\sup \left\{|f(A a)| ; f \in \mathscr{B}_{1}^{*}, a \in \mathscr{B}_{+} \cap \mathscr{B}_{1}\right\} .
$$


But since $a \in \mathscr{B}_{+}$and $A$ is positive $A a \in \mathscr{B}_{+}$. Moreover the supremum of $|f(A a)|$ over $f \in \mathscr{B}_{1}^{*}$ coincides with the supremum over $f \in \mathscr{B}_{+}^{*} \cap \mathscr{B}_{1}^{*}$. This follows from Theorem 1.3 of [2] because the norm is monotonic on $\mathscr{B}$. Therefore

$$
\begin{aligned}
\|A\|_{+} & =\sup \left\{f(A a) ; f \in \mathscr{B}_{+}^{*} \cap \mathscr{B}_{1}^{*}, a \in \mathscr{B}_{+} \cap \mathscr{B}_{1}\right\} \\
& =\sup \left\{\left(A^{*} f\right)(a) ; f \in \mathscr{B}_{+}^{*} \cap \mathscr{B}_{1}^{*}, a \in \mathscr{B}_{+} \cap \mathscr{B}_{1}\right\} \\
& \leq \sup \left\{\left(A^{*} f\right)(a) ; f \in \mathscr{B}_{+}^{*} \cap \mathscr{B}_{1}^{*}, a \in \mathscr{B}_{1}\right\} \\
& =\left\|A^{*}\right\|_{+} .
\end{aligned}
$$

But if the norm is monotonic over $\mathscr{B}^{*}$ the second of the above suprema attains the value $\left\|A^{*}\right\|_{+}$, by Theorem 1.4 of [2].

Remark. Once again monotonicity of the norm on $\mathscr{B}_{*}$ is necessary to prove $1 \Rightarrow 2$, in Theorem 1.2 , and monotonicity on $\mathscr{B}$ to prove $2 \Rightarrow 1$.

Alternative versions of Theorems 1.1 and 1.2 can be proved in special cases. For example, if $\mathscr{B}$ is equipped with the order norm $\|a\|=N(a) \bigvee N(-a)$ then Condition 2 of Theorem 1.1 is equivalent to $H$ generating a positive $C_{0}$-semigroup $S$ with $\left\|S_{t}\right\| \leq M \exp \{\omega t\}$. This follows from the proofs of Theorem 1.1 and Theorem 3.1 of [1]. It is not clear, however, if the order norm property ensures that $\left\|S_{t}\right\|=\left\|S_{t}\right\|_{+}$. We return to discussion of this point in Section 3.

In the next section we relate Theorems 1.1 and 1.2 to the corresponding Riesz norm results in [1] and discuss in further detail the relevance of monotonicity of the norm, and monotonicity of the dual norm.

\section{§2. Equivalent Riesz Norms}

We begin by examining the question of existence of an equivalent Riesz norm on an ordered Banach space. Our basic conclusion is that an ordered Banach space has an equivalent Riesz norm if, and only if, the positive cone is normal and generating. In fact the next theorem contains more detailed information. (A version of this theorem occurs in [3]).

Theorem 2.1. Let $\left(\mathscr{B}, \mathscr{B}_{+},\|\cdot\|\right)$ be an ordered Banach space, 
assume that $\mathscr{B}_{+}$is generating, and define the norm $\|\cdot\|_{r}$ by

$$
\|a\|_{r}=\inf \left\{\lambda \geq 0 ;-\lambda u \leq a \leq \lambda u, u \in \mathscr{B}_{+} \cap \mathscr{B}_{1}\right\} \text {. }
$$

It follows that $\|\cdot\|_{\text {r }}$ is a Riesz norm and

$$
\|a\|_{r}=\inf \left\{\|b+c\| ; a=b-c, b, c \in \mathscr{B}_{+}\right\} .
$$

Moreover the following conditions are equivalent:

1. $\|\cdot\|_{r}$ is equivalent to $\|\cdot\|$,

2. $\mathscr{B}_{+}$is normal.

Proof. First remark that $\|a\|_{r}$ is defined for all $a \in \mathscr{B}$ because $\mathscr{B}_{+}$ is assumed to be generating.

Second assume $-b \leq a \leq b$ and $-\lambda u \leq b \leq \lambda u$. Then $-\lambda u \leq a \leq \lambda u$ and $\|a\|_{r} \leq \lambda$. Taking the infimum over all possible $\lambda$ then gives $\|a\|_{r} \leq\|b\|_{r}$, i. e., $\|\cdot\|_{r}$ is absolutely monotone. But given $\varepsilon>0$ and $a \in \mathscr{B}$ one can choose $u \in \mathscr{B}_{+} \cap \mathscr{B}_{1}$ such that $-(1+\varepsilon)\|a\|_{r} u \leq a \leq(1+\varepsilon)$ $\|a\|_{r} u$. Hence setting $b=(1+\varepsilon)\|a\|_{r} u$ one has $-b \leq a \leq b$ and since $b \in \mathscr{B}_{+}$one also has $\|b\|_{r} \leq(1+\varepsilon)\|a\|_{r}$. Thus both Riesz norm properties are valid.

Next if $-\lambda u \leq a \leq \lambda u$ and one sets $2 b=\lambda u+a, 2 c=\lambda u-a$, then $b, c \in \mathscr{B}_{+}, a=b-c$, and $\lambda u=b+c$. Conversely if $a=b-c$ with $b, c \in \mathscr{B}_{+}$ and one sets $\lambda u=b+c$ then $-\lambda u \leq a \leq \lambda u$. Therefore the second representation of $\|\cdot\|_{r}$ is valid.

Finally consider the equivalence of the two conditions. We first remark that since $\mathscr{B}_{+}$is generating it is $\gamma$-generating for some $\gamma \geq 1$. Therefore each $a \in \mathscr{B}$ has a decomposition $a=b-c$ with $b, c \in \mathscr{B}$ and

Hence

$$
\|b\|+\|c \mid i \leq \gamma\| a \| \text {. }
$$

$$
\|a\|_{r} \leq\|b+c\| \leq\|b\|+\|c\| \leq r\|a\|_{1} .
$$

$1 \Rightarrow 2$. If $\|\cdot\|_{r}$ is equivalent to $\|\cdot\|$ there is a $\delta$ such that $\delta\|a\| \leq$ $\|a\|_{r}$ for all $a \in \mathscr{B}$. But if $0 \leq a \leq b$ then $-\lambda u \leq a \leq \lambda u$ with $\lambda=\|b\|$ and $u=b /\|b\| \in \mathscr{B}_{+} \cap \mathscr{B}_{1}$. Thus $\|a\|_{r} \leq\|b\|$. Consequently $\delta_{\|}\|a\| \leq\|b\|$ and this implies that $\mathscr{B}_{+}$is normal.

$2 \Rightarrow 1$. If $\mathscr{B}_{+}$is normal there is a $\beta \geq 1$ such that $a \leq b \leq c$ implies $\|b\| \leq \beta(\|a|| \vee\| c||)$. Therefore $-\lambda u \leq a \leq \lambda u$ implies $\|a\| \leq \beta \lambda\|u\|$ and consequently $\|a\| \leq \beta\|a\|_{r}$. But we established above that $\|a\|_{r} \leq \delta|| a \mid j$ and hence the two norms are equivalent. 
Remark. Throughout the sequel $\|\cdot\|_{r}$ will denote the Riesz norm associated with $\left(\mathscr{B}, \mathscr{B}_{+},\|\cdot\|\right)$ by the construction of Theorem 2. 1 .

Next assume $\mathscr{B}_{+}$is normal and generating and consider Theorem 3.5 of [1] applied to the equivalent system $\left(\mathscr{B}, \mathscr{B}_{+},\|\cdot\|_{r}\right)$. This theorem would imply equivalence of the two conditions of Theorem 1. 1 , applied to the system $\left(\mathscr{B}, \mathscr{B}_{+},\|\cdot\|\right)$, but for two differences. First $\left\|S_{t}\right\|_{+}$replaces the Riesz norm $\left\|S_{t}\right\|_{r}$, i. e.,

$$
\left\|S_{t}\right\|_{r}=\sup \left\{\left\|S_{t} a\right\|_{r} ;\|a\|_{r} \leq 1\right\} .
$$

Second the canonical half-norm $N$ associated with $(\mathscr{B},\|\cdot\|)$ occurs in place of the canonical half-norm $N_{r}$ associated with $\left(\mathscr{B},\|\cdot\|_{r}\right)$. Thus equivalence of the conditions of Theorem 1.1 would be a corollary of Theorem 3.5 of [1] if one had $\left\|S_{t}\right\|_{+}=|| S_{t} \|_{r}$ and also $N=N_{r}$. Hence we next analyze criteria for these equalities.

Since by Lemma 3.3 of [1] the Riesz norm of a positive operator is attained on positive elements one has $\left\|S_{t}\right\|_{+}=\left\|S_{t}\right\|_{r}$ whenever $\|a\|=$ $\|a\|_{r}$ for all $a \in \mathscr{B}_{+}$, and this latter identification is probably inevitable if one wants equality of norms for all semigroups. Thus we examine a criterion for $\|a\|=\|\left. a\right|_{r}$ whenever $a \in \mathscr{B}_{+}$.

Proposition 2.2. Let $\left(\mathscr{B}, \mathscr{B}_{+}, \mid \cdot \cdot \|\right)$ be an ordered Banach space with $\mathscr{B}+$ generating.

The following conditions are equivalent:

1. $\|a\|=\|a\|_{r}$ for all $a \in \mathscr{B}_{+}$,

2. $\|\cdot\|$ is monotone on $\mathscr{B}$.

Proof. If $a \in \mathscr{B}_{+}$and $a=b-c$ with $b, c \in \mathscr{B}_{+}$then $b=a+c \in \mathscr{B}_{+}$. So $a=(a+c)-c$ with $c \in \mathscr{B}_{+}$is the most general decomposition of $a$ into positive and negative components. Thus

$$
\|a\|_{r}=\inf \left\{\|a+c\| ; c \in \mathscr{B}_{+}\right\}=N(a),
$$

by the second representation of $\|\bullet\|_{r}$ in Theorem 2. 1. But Condition 2 is equivalent to $N(a)=\|a\|$ for all $a \in \mathscr{B}_{+}$by Theorem 2.3 of [2] and hence the conditions of the proposition are equivalent.

Next consider equality of the half-norms. 
Proposition 2.3. Let $\left(\mathscr{B}, \mathscr{B}_{+},\|\cdot\|\right)$ be an ordered Banach space with $\mathscr{B}_{+}$normal and generating. Further let $N$, and $N_{r}$, denote the canonical half-norms associated with $\left(\mathscr{B}, \mathscr{B}_{+},\|\cdot\|\right)$, and $\left(\mathscr{B}, \mathscr{B}_{+}\right.$, $\left.\|\cdot\|_{r}\right)$, respectively.

If $\|\cdot\|$ is monotone on $\mathscr{B}$ then the following conditions are equivalent:

1. $N=N_{r}$

2. $\|\cdot\|$ is monotone on $\mathscr{B}^{*}$.

Proof. Since $\|\cdot\|_{r}$ is monotone on $\mathscr{B}^{*}$ one has

$$
N_{r}(a)=\inf \left\{\|b\|_{r} ; \quad b \geq a, b \geq 0\right\}
$$

by Theorem 2.4 of [2]. But in the proof of Proposition 2.2 it was established that $\|b\|_{r}=N(b)$ for $b \geq 0$. Therefore

$$
\begin{aligned}
N_{r}(a) & =\inf \{N(b) ; b \geq a, b \geq 0\} \\
& \geq \inf \{N(b) ; b \geq a\}=N(a) . \\
1 \Rightarrow 2 . \quad \quad N(a) & =N_{r}(a) \\
& =\inf \left\{\|b\|_{r} ; b \geq a, b \geq 0\right\} \\
& =\inf \{\|b\| ; b \geq a, b \geq 0\}
\end{aligned}
$$

where the first equality follows because $\|\cdot\|_{r}$ is monotonic on $\mathscr{B}^{*}$, Theorem 2.4 of [2], and the second follows because $\|\cdot\|$ is monotonic on $\mathscr{B}$, Proposition 2.2 above. Therefore $\|\cdot\|$ is monotonic on $\mathscr{B}^{*}$ by another application of Theorem 2.4 of [2].

$2 \Rightarrow 1$. One has

$$
N(a)=\inf \left\{\| b_{\|} \mid ; b \geq a, b \geq 0\right\}
$$

by Theorem 2.4 of [2]. But $;|b| \mid \geq N(b)=\|b\|_{r}$ for $b \geq 0$ so

$$
N(a) \geq \inf \left\{\|b\|_{r} ; b \geq a, b \geq 0\right\}=N_{r}(a) .
$$

But we established above that $N_{r} \geq N$ and hence one has equality.

The moral of Propositions 2.2 and 2.3 is that if one attempts to prove a version of Theorem 1.1, with the assumption that $\mathscr{B}_{+}$is normal and generating, by construction of the equivalent Riesz norm $\|\cdot\|_{r}$ and application of Theorem 3.5 of [1], then monotonicity of the norm and dual norm appear unavoidable.

We conclude this general discussion of equivalent Riesz norms 
with criteria for comparison of $\|\cdot\|$ and $\|\cdot\|_{r}$, and with a remark on the dual Riesz norm.

Theorem 2.4. Let $\left(\mathscr{B}, \mathscr{B}_{+},\|\cdot\|\right)$ be an ordered Banach space with $\mathscr{B}_{+}$generating.

The following conditions are equivalent:

1. $\|\cdot\|$ is absolutely monotone on $\mathscr{B}$,

2. $\|a\| \leq\|a\|_{r}$ for all $a \in \mathscr{B}$.

Moreover the following conditions are equivalent:

$1 * \quad\|\cdot\|$ is absolutely monotone on $\mathscr{B}^{*}$,

2*. $\quad\|a\| \geq\|a\|_{r}$ for all $a \in \mathscr{B}$.

Proof. $1 \Rightarrow 2$. If $-\lambda u \leq a \leq \lambda u$ with $u \in \mathscr{B}_{+} \cap \mathscr{B}_{1}$ then absolute monotonicity implies $\|a\| \leq \lambda$ and hence $\|a\| \leq\|a\|_{r}$.

$2 \Rightarrow 1$. If $\|a\| \leq\|a\|_{r}$ and $-b \leq a \leq b$ then $\|a\| \leq\|a\|_{r} \leq\|b\|_{r}$ because $\|\cdot\|_{r}$ is absolutely monotone on $\mathscr{B}$. But $b \in \mathscr{B}_{+}$and hence $\|b\|_{r}=N(b)$ $\leq\|, b\|$ by the argument used in the proof of Proposition 2. 2. Therefore $\|a\| \leq\|b\|$ and $\|\cdot\|$ is absolutely monotone.

$1^{*} \Rightarrow 2^{*}$. It follows from the dual reformulation [1] of Condition $1 *$ that given $a \in \mathscr{B}$ and $\varepsilon>0$ there is a $b \in \mathscr{B}+$ such that $\|b\| \leq(1+\varepsilon)\|a\|$ and $-b \leq a \leq b$. Therefore $\|a\|_{r} \leq(1+\varepsilon)\|a\|$ and Condition $2^{*}$ follows in the limit that $\varepsilon$ tends to zero.

$2^{*} \Rightarrow 1^{*}$. First note that since $\|a\|_{r} \leq\|a\|$ the dual Riesz norm satisfies the opposite inequality

$$
\begin{aligned}
\|f\|_{r} & =\sup \left\{|f(a)| ;\|a\|_{r} \leq 1\right\} \\
& \geq \sup \{|f(a)| ;\|a\| \leq 1\}=\|f\| .
\end{aligned}
$$

Now suppose $-g \leq f \leq g$ then $\|f\|_{r} \leq\|g\|_{r}$ because $\|\cdot\|_{r}$ is a Riesz norm. Next for $\varepsilon>0$ choose $a \in \mathscr{B}$ such that

$$
\|g\|_{r}\left|a \|_{r} \leq(1+\varepsilon)\right| g(a) \mid \text {. }
$$

Hence $-\lambda u \leq a \leq \lambda u$ with $u \in \mathscr{B}_{+} \cap \mathscr{B}_{1}$ then

$$
\|g\|_{r}\|a\|_{r} \leq(1+\varepsilon) \lambda g(u) \leq(1+\varepsilon) \lambda\|g\|
$$

and one concludes that $\|g\|_{r} \leq\|g\|$. Combining these conclusions one has $\|f\| \leq\|g\|$ and so $\|\cdot\|$ is absolutely monotone on $\mathscr{B}^{*}$. 
Finally note that if $\|\cdot\|$ is a Riesz norm on $\mathscr{B}$ then the dual norm is a Riesz norm on $\mathscr{B}^{*}$ and it follows from [1] that

$$
\|f\|=\inf \left\{\lambda \geq 0 ;-\lambda g \leq f \leq \lambda g, g \in \mathscr{B}_{1}^{*}\right\} .
$$

An analogous representation is true for the dual norm $\|\cdot\|_{r}$.

Theorem 2.5. Let $\left(\mathscr{B}, \mathscr{B}_{+},\|\cdot\|\right)$ be an ordered Banach space with $\mathscr{B}_{+}$normal and generating. Let $\|\cdot\|_{r}$ denote the equivalent Riesz norm, and the associated dual norm, i.e.,

$$
\|f\|_{r}=\sup \left\{|f(a)| ;\|a\|_{r} \leq 1\right\}
$$

for all $f \in \mathscr{B}^{*}$.

It follows that

$$
\begin{aligned}
\|f\|_{r} & =\inf \left\{\lambda \geq 0 ; \quad-\lambda g \leq f \leq \lambda g, g \in \mathscr{B}_{1}^{*}\right\} \\
& =\inf \left\{\|g+h\| ; f=g-h, g, h \in \mathscr{B}_{+}\right\} .
\end{aligned}
$$

Proof. First define

$$
\|f\|^{\prime}=\inf \left\{\lambda \geq 0 ; \quad-\lambda g \leq f \leq \lambda g, g \in \mathscr{B}_{1}^{*}\right\} .
$$

Now if $-\mu b \leq a \leq \mu b$ for $b \in \mathscr{B}_{1}$ and $-\lambda g \leq f \leq \lambda g$ for $g \in \mathscr{B}_{1}^{*}$ then $|f(a)| \leq \lambda \mu g(b) \leq \lambda \mu$. Taking the infimum over $\mu$ gives $|f(a)| \leq \lambda|| a \|_{r}$ and hence $\|f\|_{r} \leq\|f\|^{\prime}$.

Next suppose $\|f\|_{r} \leq 1$ and define $p$ by

$$
p(a)=\inf \left\{\|a+b+c\|+f(b-c) ; b, c \in \mathscr{B}_{+}\right\}
$$

for $a \in \mathscr{B}$. It follows that $p$ is subadditive, positively homogeneous, and $p(a) \leq\|a\|$. But setting $a=b^{\prime}-c^{\prime}$ with $b^{\prime}, c^{\prime} \in \mathscr{B}_{+}$one has

$$
-\left(a^{\prime}+b+c\right) \leq a+b-c \leq a^{\prime}+b+c
$$

where $a^{\prime}=b^{\prime}+c^{\prime}$. Hence

Therefore

$$
\|a+b-c\|_{r} \leq\left\|a^{\prime}+b+c\right\| .
$$

$$
\begin{aligned}
|f(b-c)| & \leq|f(a)|+|f(a+b-c)| \\
& \leq|f(a)|+\left\|a^{\prime}+b+c\right\| \\
& \leq|f(a)|+\|a+b+c||+2\| c^{\prime}|| .
\end{aligned}
$$

Consequently

$$
\begin{gathered}
p(a) \geq-f(a)-2 \| c^{\prime} \mid, \\
\geq-k\|a\|
\end{gathered}
$$


for some finite $k$. Thus, by the Hahn-Banach theorem, there exists a linear functional $g$ such that $g(a) \leq p(a)$ for all $a \in \mathscr{B}$. Therefore $\pm g(a)=g( \pm a) \leq\|a\|$ and $g$ is continuous with $\|g\| \leq 1$. But if $-a \in \mathscr{B}_{+}$ then setting $b=a$ and $c=0$ one finds $p(a) \leq f(a)$, and setting $b=0$ and $c=a$ one has $p(a) \leq-f(a)$. Thus

$$
g(-a) \leq-|f(a)|
$$

or, equivalently,

$$
-g(a) \leq f(a) \leq g(a)
$$

Therefore $\|f\|^{\prime} \leq 1=\|f\|_{r}$.

Combination of the above conclusions gives $\|f\|_{r}=\|f\|^{\prime}$. The second representation of $\|f\|_{r}$ follows as in the proof of a similar relation in Theorem 2. 1.

\section{§3. Concluding Remarks}

The major difference between Theorem 1.1 and Theorem 3.5 of [1] is the replacement of $\left\|S_{t}\right\|$ by $\left\|S_{t}\right\|_{+}$and it is of interest to investigate circumstances under which these norms coincide. If $\left(\mathscr{B}, \mathscr{B}_{+},\|\cdot\|\right)$ is a Riesz norm space then $\|A\|=\|A\|_{+}$for all positive $A \in \mathfrak{B}(\mathscr{B})$ by Lemma 3.3 of [1] but the Riesz norm property is possibly unnecessary for this identification. If $\mathscr{B}$ is equipped with the order norm, $\|a\|=N(a) \vee N(-a)$, and the dual norm is monotone on $\mathscr{B}^{*}$, then one also has this equality. This is established by first remarking that since $\mathscr{B}$ has the order norm each $f \in \mathscr{B}^{*}$ has a Jordan decomposition, [4] [5], i. e., there are $g, h \in \mathscr{B}_{+}^{*}$ such that $f=g-h$ and

$$
\|f\|=\|g\|+\|h\|
$$

Therefore

$$
\begin{aligned}
\left\|A^{*} f\right\| & \leq\left\|A^{*} g\right\|+\left\|A^{*} h\right\| \\
& \leq\left\|A^{*}\right\|_{+}\|g\|+\left\|A^{*}\right\|_{+}\|h\|=\left\|A^{*}\right\|_{+}\|f\|
\end{aligned}
$$

which gives $\left\|A^{*}\right\| \leq \|\left. A^{*}\right|_{1+}$. But the converse inequality is always true and hence $\|A\|=\left\|A^{*}\right\|=\left\|A^{*}\right\|_{+}$. The order norm is, however, monotonic and hence monotonicity of the norm on $\mathscr{B}^{*}$ suffices to establish $\|A\|=\|A\|_{+}$, by Lemma 1.3 .

Conversely norm monotonicity appears necessary to ensure that a 
positive operator attains its norm on the positive elements. It is easy to construct an example with a non-monotonic norm and with a positive contraction semigroup for which $\left\|S_{t}\right\|_{+}<\left\|S_{t}\right\|$. Let $\mathscr{B}=L^{2}(0, \pi)$ with $\mathscr{B}_{+}$the positive $L^{2}$-functions and

$$
\|f\|=\left(\|f\|_{2}^{2}+\|D f\|_{2}^{2}\right)^{1 / 2}
$$

where $D$ denotes the closed differentiation operator without boundary conditions. Then $H=-D^{*} D$ is the self-adjoint double-differentiation operator with Neumann boundary conditions. Thus if $S_{t}=\exp \{-t H / 2\}$ then

$$
\left\|S_{t} f\right\|^{2}=\left(f,(1+H) S_{2 t} f\right)
$$

and $\left\|S_{1}\right\|$ is attained uniquely on the non-positive function $f ; f(x)$ $=(2 / \pi)^{1 / 2} \cos x$.

It would be of interest to establish further links between monotonicity of the norm on $\mathscr{B}$, and $\mathscr{B}^{*}$, and the equality $\|A\|=\|A\|_{+}$ for positive $A$. It would also be cf interest to decide whether the norm dual to the order norm is absolutely monotone, or monotone. This is certainly the case $[1]$ if $\left(\mathscr{B}, \mathscr{B}_{+},\|\cdot\|\right)$ has an identity.

\section{Acknowledgements}

I am indebted to Charles Batty for the proof of Theorem 2.5. It improves a weaker result contained in an earlier version of this paper.

\section{References}

[1] Robinson, D. W., Continuous semigroups on ordered Banach spaces. J. Funct. An., 51 (1983), 268-284.

[2] Robinson, D. W. and Yamamuro, S., The canonical half-norm dual half-norms, and monotonic norms. Tôhoku Math. Journ, 35 (1983), 375-386.

[3] Davies, E. B., The structure and ideal theory of the predual of a Banach lattice, Trans. Amer. Math. Soc., 131 (1968), 544-555.

[4] Grosberg, J. and Krein, M., Sur la décomposition des fonctionelles en composantes positives, C. R. (Doklady) Acad. Sci. URSS (N.S) 25 (1939), 723-726.

[5] Robinson, D. W. and Yamamuro, S., The Jordan decomposition and half-norms. Pac. J. Math, to appear 